\title{
Relevancia de las referencias bibliográficas en artículos de revistas médicas
}

\author{
MANUEL OYARZÚN G.**** y MARCELA AGUIRRE C.***
}

\author{
$\underline{\text { Relevance of bibliographic references in articles published in medical journals }}$
}

Bibliographic references are an important constituent of all scientific papers. The authors are responsible for their accuracy and they should follow the international requirements of style for references, adopted by the journal in which their paper is going to be submitted. Revista Chilena de Enfermedades Respiratorias follows the "Uniform Requirements" established by the Vancouver Group of Medical Editors. Authors should do a suitable selection of references, providing direct citations to original research sources, close related to the content of the paper. The most common errors in reference list are: a) an excessive number of irrelevant citations; b) the omission of important articles; c) a wrong claim of priority in reporting an outcome. It is recommended avoid using abstracts as references, unless they have been published in the last three years. The use of electronic references should indicate: "available in", web site and the access date. International visibility of local journals from developing countries can be improved by including as references previous reports published in these national journals.

Key words: Bibliographic references, medical journals, medical manuscripts, Internet, publications.

\section{Resumen}

Las referencias bibliográficas son un componente importante de todos los manuscritos científicos. Los autores son responsables de la exactitud de las referencias y deben seguir los requerimientos internacionales de estilo adoptados por la revista a la cual el manuscrito será enviado a publicación. La Revista Chilena de Enfermedades Respiratorias sigue los “Requerimientos Uniformes” establecido por el Grupo de Editores Médicos de Vancouver. Los autores deben hacer una adecuada selección de las referencias, proporcionando citas directas de las fuentes originales de investigación que tengan estrecha relación con los contenidos del manuscrito. Los errores más comunes en las referencias son: a) excesivo número de referencias irrelevantes; b) omisión de artículos importantes; c) declaración de prioridad errónea en comunicar un resultado. Se recomienda evitar usar resúmenes de congresos como referencias, a menos que hayan sido publicados en los últimos tres años. Al usar referencias electrónicas se debe indicar: "disponible en", el sitio web y la fecha de acceso. En los países en desarrollo la visibilidad internacional de sus revistas puede mejorar si se incluyen como referencias comunicaciones previas publicadas en estas revistas nacionales.

Palabras clave: Referencias bibliográficas, revistas médicas, manuscritos médicos, Internet, publicaciones.

La sección bibliografía o de referencias bibliográficas habitualmente se encuentra al final del texto de los artículos publicados en las revistas científicas biomédicas y aunque está en esa ubicación extrema es un componente esencial de un artículo como cualquier otra sección, a tal punto que constituye un índice de la prolijidad, pertinencia, actualidad y acuciosidad con la que el artículo fue concebido y luego escrito. Las referencias bibliográficas proporcionan la sus-

\footnotetext{
* Editor, Revista Chilena de Enfermedades Respiratorias.

** Programa de Fisiopatología, Instituto de Ciencias Biomédicas, Facultad de Medicina, Universidad de Chile. Santiago-Chile.

*** Programa de Información Científica, CONICyT, Santiago-Chile.
} 
tentación de la génesis, propósito, metodología e interpretación del trabajo realizado, es decir, refleja que toda investigación debe dar crédito al aporte realizado por quienes los precedieron en publicar estudios similares o relacionados ${ }^{1}$. Existen diferentes reglas o normas para las citas en el texto del artículo y referencias bibliográficas al final del artículo, dependiendo de la especialidad y de la norma o estilo de la revista.

El proceso de creación del conocimiento ha sido comparado con la construcción de una estructura en que cada autor contribuye en algún grado a la estructura final constituida por la comprobación de una idea, concepto o principio, que idealmente formará parte del conocimiento humano sobre el tema abordado.

Se ha señalado la imposibilidad de escribir ingeniosamente sobre cualquier tema en medicina sin conocer lo que ya ha sido escrito sobre esa materia ${ }^{2}$.

Las referencias bibliográficas deben ser precisas para evitar que los lectores y bibliotecólogos malgasten su tiempo en la búsqueda de un artículo citado incorrectamente. La citación de una referencia significa que esta ha sido leída por quienes firman el artículo. No es una buena práctica copiar una referencia de una bibliografía de otro artículo sin verificar su procedencia y contenido ${ }^{3}$.

Se han descrito diversos errores que desaconsejan esta práctica que algunos autores aplican intentando ahorrar tiempo en la preparación de manuscritos. Se ha dado el caso que una referencia citada en 6 artículos publicados en el mismo número de la misma revista (Journal of Geophysical Research del 21 de septiembre de 1972) estuvo correctamente citada en cuanto a señalar los números correspondientes al volumen y páginas inicial y final en sólo 2 de los 6 artículos ${ }^{3}$. Otros casos emblemáticos -mencionados por el Dr. Julius H. Comroe, Jr. en su curso "Scientific Writing" dictado en 1974 en la Universidad de California, San Francisco, han sido citaciones erróneas del nombre del autor de un artículo, que se han mantenido por muchos años a partir de una citación inicial defectuosa y que ulteriormente ha sido copiada por autores que han continuado publicando sobre el mismo tema. Un ejemplo de este tipo de errores afectó al Dr. Martín Rushton quien en 1946 firmó un artículo de su autoría en el British Dental Journal agregando a continuación de su nombre las siglas MB, ChB, Cantab, que indicaban sus grados académicos y la universidad en que los obtuvo: Cantab (abreviatura de Cantabrigiensis) corresponde a Universidad de Cambridge. Un autor estadounidense interpretó erróneamente estas siglas y desde entonces el artículo fue citado equivocadamente, atribuyendo su autoría a dos autores Rushton M y Cantab $\mathrm{MB}^{(\mathrm{i} !)}$.

Las normas de publicación de las revistas establecen que la exactitud de las referencias bibliográficas es responsabilidad de los autores. Sin embargo, cada vez es más frecuente que las oficinas editoriales de las revistas verifiquen la exactitud de todas las referencias de sus artículos. Como esta práctica aun no es universal, es posible que la versión final de un artículo ya publicado contenga errores en sus referencias bibliográficas. Para verificar la exactitud de sus referencias se recomienda a los autores usar una fuente bibliográfica electrónica confiable como PubMed o copias impresas de las referencias originales ${ }^{4}$.

Las revistas biomédicas exigen uniformidad en el formato de los artículos que reciben para su eventual publicación. Estas exigencias están especificadas en las "normas de publicación para los autores" y dentro de ellas se establecen las normas para citar las referencias bibliográficas. La Revista Chilena de Enfermedades Respiratorias sigue las recomendaciones del así llamado "Grupo de Vancouver" adoptadas también por la Asociación Mundial de Editores de Revistas Médicas (WAME: World Association of Medical Editors $)^{4}$. Los editores debemos hacer respetar estas normas de publicación ${ }^{4,5}$ que son razonables y relativamente fáciles de cumplir, siendo del todo aconsejable que los autores tomen conocimiento de estas normas antes de empezar a escribir su manuscrito. Las normas de Vancouver utilizan el término referencias bibliográficas para aludir a las citas bibliográficas enumeradas en el texto por números arábigos consecutivos y enlistadas según su orden de aparición.

En la experiencia del Comité Editorial de esta revista es infrecuente que los autores cumplan plenamente las normas de publicación. Habitualmente los problemas en el seguimiento de las normas del formato se concentran en la sección de referencias bibliográficas de los manuscritos recibidos.

El autor de una tesis de postgrado está obligado a demostrar a través de la extensa bibliografía de este documento, que ha buscado, leído y evaluado la "literatura" en el campo que abarca su tesis y que sabe cómo hacerlo académicamente. Por su parte el autor de un manuscrito de un trabajo original enviado a publicación a una revista con revisión de pares, si bien también debe buscar, leer y sopesar la "literatura" pertinente, no da más pruebas de lo que ha leído o consultado 
que los artículos que ha citado en la acotada lista de referencias de su manuscrito. Por lo tanto, la lista de referencias bibliográficas en cuanto a su calidad, pertinencia y actualidad, es un índice del buen criterio, selectividad y moderación con el que los autores han logrado filtrar la información trivial de la pertinente.

En general los artículos de revisión constituyen una forma de orientar al lector sobre la información bibliográfica que se dispone sobre un tema específico. Sin embargo, no siempre reflejan el trabajo original en forma adecuada. Por lo tanto, se recomienda a los autores de estos artículos que en lo posible proporcionen las referencias bibliográficas de las fuentes del trabajo original y especialmente que citen los artículos que son claves en el desarrollo del conocimiento alcanzado en el tema ${ }^{4}$.

Entre las recomendaciones generales sobre referencias bibliográficas del Comité Internacional de Editores de Revistas Médicas está evitar la cita de una "comunicación personal" a menos que ésta proporcione una información esencial no disponible en una fuente de acceso público. Para hacerlo se debe obtener permiso escrito y confirmar la exactitud de la fuente. La referencia a la información contenida en un manuscrito aceptado, pero aún no publicado debe ser rotulada como "en prensa". La información de un manuscrito enviado a publicación, pero aún no aceptado debe ser señalado como "observaciones no publicadas", en todos estos casos se debe obtener permiso escrito de la fuente.

Un caso especial lo constituye la citación de resúmenes presentados a congresos ("abstracts") como referencias bibliográficas. El ya citado Comité Internacional de Editores, recomienda evitar esta práctica, aunque no la proscribe ${ }^{4}$. Diversos estudios en ciencias médicas y disciplinas relacionadas han demostrado que entre 30 a $50 \%$ de los resúmenes presentados en congresos anuales de las sociedades que cubren estas disciplinas se materializan en una publicación in extenso en los siguientes cinco años post-congreso ${ }^{6}$. Por lo tanto, los "abstracts" deben considerarse como publicaciones preliminares y no es recomendable incluirlos como citas bibliográficas, a menos que el resumen haya sido publicado recientemente, es decir, en los últimos tres años en una revista con sistema de revisión por pares, de acceso universal, con información original ${ }^{6}$. Al respecto hay que hacer notar que los resúmenes publicados en la Revista Chilena de Enfermedades Respiratorias dejaron de ser indexados en SciELO-Chile a contar del año 2009 y que la National Library of Medicine (NLM) de EE.UU. tampoco incluye en MEDLINE los "abstracts" que se presentan en reuniones anuales de una especialidad y que se publican en un número completo o suplemento de sus revistas indexadas.

Un comentario especial merece la inclusión de referencias bibliográficas electrónicas en artículos biomédicos. Los documentos electrónicos en línea están disponibles desde fines de 1995 con la aparición de Internet. Los elementos que los identifican universalmente son disponibilidad o acceso, sitio web, fecha de consulta y fecha de actualización. Para artículos en formato electrónico se han elaborado normas o guías. La citación completa debe incluir: nombres de los autores, título del documento, fecha de publicación o actualización, seguida del sitio electrónico y fecha en que se hizo la consulta. A los autores se les debe insistir en que proporcionen toda esta información, verificando que la dirección del sitio web es correcta para lograr ubicar sin dificultad sus citas electrónicas en Internet. Un análisis de referencias electrónicas realizado en el volumen 132 de la Revista Médica de Chile correspondiente al año 2004, concluyó que más del $60 \%$ de las referencias electrónicas estaban incompletas en su construcción y que sólo la mitad de ellas indicaba la fecha de consulta ${ }^{7}$.

En los últimos años la cita a documentos electrónicos en biomedicina ha aumentado considerablemente, como consecuencia de la disponibilidad de bases de datos en línea en $w w w$ y ediciones electrónicas de revistas científicas, que permiten por medio de búsquedas rápidas, especializadas, avanzadas y eficientes, recuperar y acceder a literatura médica, que en muchos casos dispone de acceso al texto completo de los artículos libremente, conociendo si dicha literatura ha sido citada o no, por ejemplo, la base de datos internacionalmente reconocida PubMed, desde el año 1997 está disponible en Internet ${ }^{8}$ en modalidad de acceso abierto.

Entre las referencias a documentos electrónicos también se debe considerar aquellas a artículos que incluyen un identificador de objeto digital, en inglés Digital Object Identifier, comúnmente conocido como DOI y artículos publicados "Ahead of Print", ambos casos siguen normativas recomendadas por el ICMJE, Comité Internacional de Editores de Revistas Médicas, señalado anteriormente en este artículo.

Lo que motiva a los editores a ser más rigurosos con los autores que presentan artículos en revistas médicas, incentivándolos a ser más cuidadosos y respetuosos de las normas y formas de citar las referencias bibliográficas en sus trabajos.

A los problemas formales que pueden tener 
las referencias bibliográficas, se han detectado y analizado los problemas de fondo que se pueden descubrir en estas citas y que son de total responsabilidad de los autores ${ }^{1}$. Las más frecuentes son las siguientes ${ }^{1}$ :

a) Exceso de referencias. Se deben escoger publicaciones originales. Los artículos de revisión que repiten resultados de otros trabajos sólo justifican su citación si en sus comentarios aportan ideas novedosas en la interpretación de resultados comunicados en artículos originales. Para paliar el exceso de referencias, habitualmente las normas de publicación para los autores limita el número de citas bibliográficas. En la Revista Chilena de Enfermedades Respiratorias se recomienda no citar más de 30 referencias en los manuscritos enviados a publicación. La lista de referencias bibliográficas debe contener sólo las referencias enumeradas en el texto, en las tablas y en las leyendas de las figuras y no otras adicionales que no hayan sido previamente enumeradas en el manuscrito.

b) Omisión de referencias. La omisión de referencias bibliográficas importantes lleva a pensar que la revisión bibliográfica que hicieron los autores fue superficial. Por otra parte, es altamente recomendable citar las referencias nacionales, no sólo porque implica reconocimiento y consideración por los autores y revistas nacionales que han investigado sobre el mismo tema, sino porque aumenta su difusión internacional y porque otorga una posibilidad de mejorar los índices bibliométricos de las revistas nacionales, como citaciones y el "factor de impacto", así como otros de igual o mayor importancia ${ }^{9}$. En este sentido es muy recomendable que los revisores y editores se preocupen que los autores citen aquellos artículos que se han publicado previamente en la misma revista nacional a la cual están enviando su manuscrito, aunque igualmente se debe cuidar de un elevado porcentaje de autocitas.

c) Autoasignación de prioridad cronológica. En algunos manuscritos sus autores expresan en el texto que son los primeros en publicar sobre el tema o sobre un tópico de este ya sea en el país, la región o en el mundo. Estas aseveraciones son difíciles de comprobar y desafortunadamente con más frecuencia de lo que pudiese esperarse, algunos editores han tenido que publicar aclaraciones en cartas al editor por solicitud de autores que han publicado previamente sobre el tema en otras revistas, o lo que es más bochornoso aún en la misma revista. Ya en 1974 el editor del New
England Journal of Medicine decidió proscribir que en el título o en el texto de los artículos publicados en esa prestigiosa revista, se aseverara que se trataba de la primera publicación sobre un determinado tema o que la condición relatada no había sido previamente descrita. Esta fue una medida de "autoprotección editorial" ya que según dicho editor en 9 de 10 veces la auto-asignación de prioridad cronológica provoca el envío de airadas cartas de reclamo negando tal prioridad ${ }^{10}$. Con fines más amplios y especialmente cuando se trata de artículos de revisión se ha propuesto especificar los criterios con los que se hizo la búsqueda bibliográfica, comunicando los índices bibliográficos consultados y el período y el o los idiomas que abarcó la búsqueda bibliográfica.

Otros errores en las listas de referencias bibliográficas de los manuscritos son la ausencia absoluta de referencias, las referencias enlistadas están desactualizadas, las referencias no corresponden a lo que se describe en el texto, las referencias son inaccesibles, referencias presentadas en distinto formato, referencias electrónicas que no conducen a los documentos referenciados por incluir vínculos inexactos o incompletos, $\mathrm{y}$ en algunos casos, referencias duplicadas. Los errores descritos pueden llegar a determinar en el proceso editorial el rechazo del manuscrito por los pares revisores.

Podemos concluir que las referencias bibliográficas de los artículos publicados en las revistas médicas tienen un altísimo grado de connotación y relevancia, contribuyendo decisivamente a tejer la red del conocimiento médico y a su admirable y desbordante crecimiento. En consecuencia, las referencias merecen una atención y dedicación especial por parte de los autores al momento de citar y listar los documentos consultados en la elaboración del manuscrito, aplicando aspectos éticos, de integridad intelectual ${ }^{11}$ y respetando las normas y formatos establecidos por las revistas, lo que finalmente se traduce en una buena práctica científica, proporcionando a los lectores fuentes confiables para acceso al conocimiento científico en un tema de interés particular.

\section{Referencias bibliográficas}

1.- REYES H. Las referencias en artículos publicados en revistas biomédicas. Rev Med Chile 2001; 129: 343-5.

2.- BEATTY W K. Searching the literature comes before writing the literature. Ann Intern Med 1973; 79: 917-24.

3.- BLANCHARD D C. References and unreferences. Science 1974; 85:1003.

4.- INTERNATIONAL COMMITTEE OF MEDICAL 
JOURNAL EDITORS. Uniform requirements for manuscripts submitted to biomedical journals: Writing and editing for biomedical publication (Updated April 2010). Disponible en: http://www.icmje.org [Consulta: 9 de abril de 2012].

5.- Normas de publicación para los autores. Rev Chil Enf Respir 2012; 28: 78-9.

6.- REYES H, ANDRESEN M, PALMA J. La importancia y el destino de los resúmenes presentados a reuniones científicas. Rev Med Chile 2011; 139: 7-10.

7.- SAGUA H. La referencia electrónica en artículos científicos. Rev Med Chile 2007; 135: 939-41.

8.- FALAGAS M E, PITSOUNI E I, MALIETZIS G A, PAPPAS G. Comparison of PubMed, Scopus, Web of Science, and Google Scholar: strengths and weaknesses.
FASEB Journal [Internet] 2008, 2: 338-42. Disponible en: http://www.fasebj.org/content/22/2/338.full. pdf + html [Consulta: 30 de abril de 2012].

9.- AGUIRRE M, OYARZÚN M. Siete años de la Revista Chilena de Enfermedades Respiratorias en SciELO: Desafíos y oportunidades. Rev Chil Enf Respir 2009; 25: 5-7.

10.- INGELFINGER F J. Why the "first report" is unacceptable? N Engl J Med 1974; 290: 740-1.

11.- BRKIĆ S, VUĖENOVIĆ M, ĐOKIĆ, Z. Title, abstract, key words and references in biomedical articles. Arch Oncology [Internet] 2002, 3: 208-9. Disponible en: http://www.doiserbia.nb.rs/img/doi/03547310/2003/0354-73100303207B.pdf [Consulta 30 de abril de 2012].

Correspondencia a:

Dr. Manuel Oyarzún G.

Programa de Fisiopatología, ICBM

Facultad de Medicina,

Universidad de Chile

E-mail: moyarzun@med.uchile.cl 